\title{
First Described Case of Gastric Volvulus Following Open Cholecystectomy
}

\author{
Sindi Diko a, c, Mohammed Elassa ${ }^{\mathrm{b}}$, Caitlin Russella, \\ Jamshed Zuberi ${ }^{\mathrm{a}}$, Derick Christian ${ }^{\mathrm{a}}$
}

\begin{abstract}
Gastric volvulus is a rare entity with the potential to become a surgical emergency if vital vascular supply is compromised. We present a case of a patient who underwent laparoscopic cholecystectomy converted to open; and after multiple admissions for intractable vomiting, she was found to have a secondary gastric volvulus during subsequent exploratory laparotomy. Due to dense adhesions, complete detorsion was deemed unsafe for the patient and a gastrojejunal bypass was created with subsequent complete resolution of symptoms. Patient has been doing well in follow-up visits.
\end{abstract}

Keywords: Open cholecystectomy; Secondary; Gastric volvulus; Postoperative complication

\section{Introduction}

Gastric volvulus is defined as an abnormal rotation of the stomach around itself; the phenomenon was first described in 1886 by Berti during an autopsy, and first treated surgically in 1897 by Berg [1]. Gastric volvulus can be classified using the Singleton system as organoaxial or mesenteroaxial depending on the axis of rotation. An organoaxial volvulus occurs when the rotation occurs along the axis connecting the stomach cardia and pylorus; whereas mesenteroaxial volvulus occurs when rotation occurs perpendicular to the long axis of the stomach, connecting the greater and lesser curvatures [2]. It can also be classified as primary or secondary: primary when the cause of rotation is attributed to the laxity of the stomach's ligamentous attachments, and secondary when there are attributable conditions such as hernias, connective tissue disorders, adhesions, or abdominal wall defects [3]. Clinical presentations vary and are

Manuscript submitted March 12, 2020, accepted March 28, 2020

aDepartment of Surgery, St. Joseph's University Medical Center, Paterson, NJ, USA

${ }^{b}$ Rowan School of Osteopathic Medicine, Stratford, NJ, USA

${ }^{\mathrm{c} C}$ Corresponding Author: Sindi Diko, Department of General Surgery, St. Joseph's University Medical Center, 703 Main St, Paterson, NJ 07503, USA. Email: Sdiko26@gmail.com

doi: https://doi.org/10.14740/jcs403 often nonspecific with vomiting and epigastric pain being most common. Diagnosis is commonly made using barium contrast study, upper gastrointestinal endoscopy, or computed tomography $(\mathrm{CT})$. Definitive management is surgery with prompt reduction of the volvulus to prevent progression into ischemia. Here, we present a rare postoperative complication, an acute secondary gastric volvulus due to postoperative adhesions in a young woman who underwent an open cholecystectomy. Of note, to our knowledge, this is the first and only description of gastric volvulus secondary to a cholecystectomy.

\section{Case Report}

\section{Investigations}

A 21-year-old Hispanic female presented with complaints of intermittent right upper quadrant abdominal pain and associated nausea and vomiting for 2 months. At the time of initial presentation, an abdominal ultrasound was consistent with a stone in the neck of the gallbladder, edematous and thickened wall at $8 \mathrm{~mm}$, and common bile duct of $5 \mathrm{~mm}$ with surrounding pericholecystic fluid. A recommendation was made for a cholecystectomy; however, the patient refused and preferred to be sent home on antibiotics. She returned 2 days later with worsening symptoms and found to be febrile and have a leukocytosis. The patient was taken to the operating room for laparoscopic cholecystectomy where she was found to have a gangrenous necrotic gallbladder with thick inflammatory rind and edema. The decision to convert to open was made as a result of difficulty in obtaining the critical view of safety secondary to the friability of the gangrenous gallbladder wall. Over the next few days the patient improved clinically and was discharged home in stable condition.

\section{Diagnosis}

Her postoperative course was complicated by intractable nausea, vomiting, and epigastric pain despite antiemetic and pain medication. After her first visit to the emergency department (ED) 2 weeks following the cholecystectomy, she underwent a series of tests including: CT imaging of her abdomen and pelvis which showed postoperative changes consistent with cholecystectomy, a dilated stomach (Fig. 1), and an inciden- 


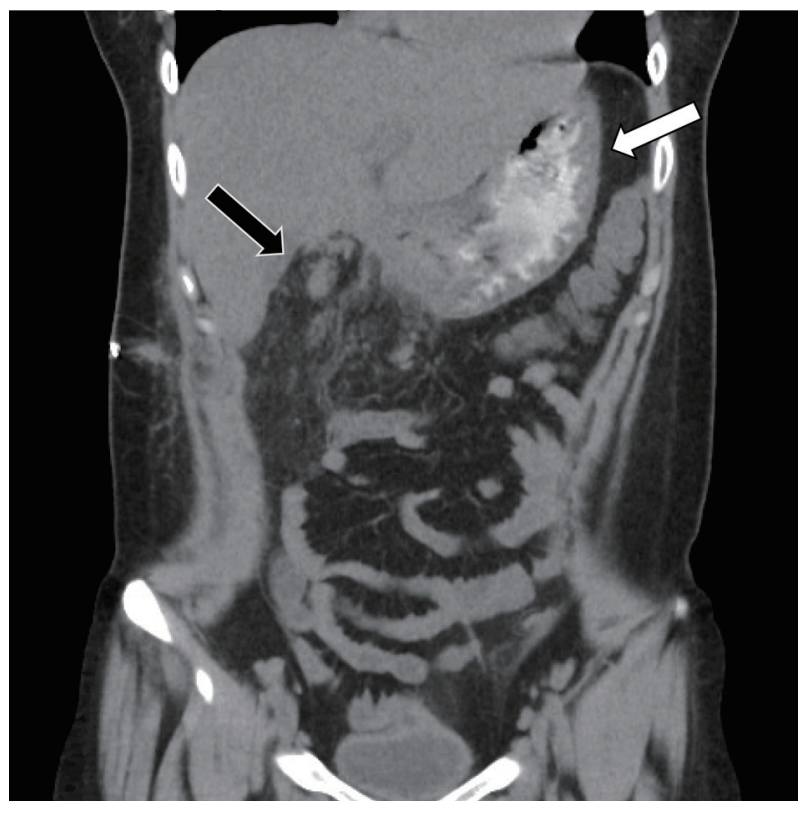

Figure 1. CT abdomen/pelvis showing no evidence of gastric volvulus with gastric distention. CT: computed tomography.

tal liver mass measuring $4.3 \times 5 \times 4.5 \mathrm{~cm}$ that was consistent with focal nodular hyperplasia (FNH); a hepatobiliary iminodiacetic acid (HIDA) scan which was negative for any biliary leak; an upper gastrointestinal series X-ray showed significant pyloric stenosis (Fig. 2), and endoscopy which demonstrated extrinsic compression of the gastric antrum with acute angulation contributing to severe obstruction of the pyloric lumen. A rheumatologic workup was conducted which resulted in a diagnosis of systemic lupus erythematosus after an elevated anti-double stranded DNA and elevation of anti-Smith antibodies were noted on labs. She was treated with a 5-day course of steroids showing clinical improvement and discharged home.

Following discharge, the patient developed intractable nausea and vomiting once again, causing her returning to the ED. This time she was found to have transaminitis. Initially, a magnetic resonance cholangiopancreatography (MRCP) showed inflammatory changes in the right upper abdomen adjacent to the pancreatic head, as well as intrahepatic biliary ductal and common bile duct dilatation with areas of narrowing. A follow-up endoscopic retrograde cholangiopancreatography (ERCP) conducted for evaluation of possible primary sclerosis cholangitis was aborted due to the endoscope being unable to traverse the stenotic pylorus. At this point, a plan was created to have a joint procedure where a diagnostic laparoscopy would be conducted in addition to an intraoperative ERCP with possible gastric stent placement.

\section{Treatment}

Intraoperatively, esophagogastroduodenoscopy (EGD) again confirmed severe obstruction, and it was evident stent placement would not be possible. Diagnostic laparoscopy demon-

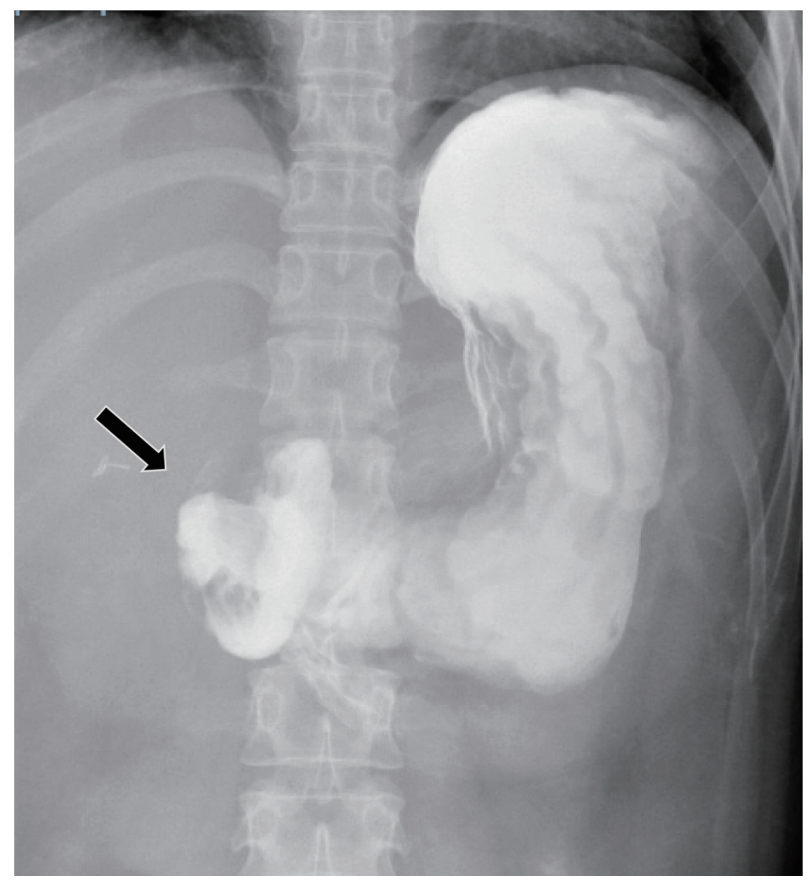

Figure 2. Upper gastrointestinal series showing evidence of significant pyloric obstruction (arrow indicating obstruction).

strated an acute gastric volvulus due to significant adhesions from the stomach to the liver bed, transverse colon and gallbladder fossa. The procedure was converted to an exploratory laparotomy after attempts to reduce laparoscopically were unsuccessful. The patient was noted to have acutely inflamed adhesions, and it was deemed unsafe to fully dissect them free from the liver bed. At that time, a gastrojejunal bypass was established to bypass the pyloric stenosis.

\section{Outcomes and follow-up}

Postoperatively, she developed a right anterior abdominal collection which was subsequently drained by interventional radiology. The remainder of her course was uneventful. She was discharged on Flagyl. She has had a number of follow-up office visits where she is improving and no longer experiencing the intractable nausea and vomiting.

\section{Discussion}

Due to its rarity, an accurate incidence of gastric volvulus is unavailable. There is a bimodal age distribution for the condition, most commonly occurring in adults after the age of 50 years old followed by children during the first year of life [4]. There is not a clear predilection for a specific gender or race. Risk factors associated with the development of volvulus are increased age, physical diaphragmatic abnormalities and phrenic nerve paralysis. Secondary gastric volvulus makes up $66 \%$ of patients, with the most common reported contributing pathology being a paraesophageal hernia [5]. 
The nonspecific presentation contributes greatly to the delayed diagnosis of gastric volvulus. The most common symptoms are postprandial epigastric discomfort, vomiting, dysphagia, difficulty belching, gastric reflux, abdominal pain, and distension. The severity of presentation is variable depending on both the degree of rotation, as well as the acuity of the volvulus. Associated with up to $70 \%$ of diagnosed volvulus is the Brochardt's triad, which is defined as pain, retching, and inability to pass a nasogastric tube [3]. With chronic volvulus, there is a risk for bleeding from gastric ulceration development leading to anemia.

Although there is no established gold standard for diagnosis of suspected gastric volvulus, there are a number of imaging modalities that prove to be useful in diagnosis. The diagnosis can be made using a barium contrast study, upper gastrointestinal endoscopy, or a CT scan. A study of 21 patients found to have acute gastric volvulus looked at the diagnostic yield of the above-mentioned imaging studies, and it was reported that barium contrast study had the highest diagnostic yield of approximately $81.3 \%$ followed by upper gastrointestinal endoscopy at $73.3 \%[6]$.

Initial management of gastric volvulus is with intravenous fluid resuscitation and placement of a nasogastric tube to facilitate immediate gastric decompression. A surgical consultation is imperative in cases where rotation occurs to point where there is considerable risk for vascular compromise. There is an increasing role for endoscopy in the management of volvulus. It allows for gastric decompression in cases where nasogastric tube insertion proves to be difficult due to degree of volvulus, provides visualization of the gastric mucosa for assessment of gastric ischemia, and can be a less invasive option for treatment of volvulus as it has been reported to be successfully utilized in detorsion of gastric volvulus [7]. In cases where endoscopy is not successful, definitive management of gastric volvulus is most commonly open surgical reduction with or without gastropexy [8]. In the case of our patient, extensive lysis of adhesions was also employed as it was the cause of the volvulus. Similarly, intraoperative decisions must be made to ensure that any contributing pathology to the volvulus is addressed and repaired to prevent recurrence following reduction. Due to the risk associated with an open surgical approach, a more conservative approach has been employed using laparoscopy for elderly patients or patients who present with chronic volvulus, and are with a lower risk for vascular compromise.

Learning points from this case include: 1) Patients with highest predilection for developing a gastric volvulus have a concurrent history of paraesophageal hernia; 2) Most cases have been reported in patients in the extremes of age groups, without any specific predilection attributed to gender; 3) Common associated presenting symptoms of gastric volvulus are postprandial epigastric discomfort, vomiting, dysphagia, reflux, and distention; 4) Brochardt's triad which consists of pain, retching, and inability to pass a nasogastric tube has been recorded in a majority of patients found to have gastric volvulus; 5) Highest diagnostic yield imaging study has been reported to be barium contrast study followed by CT; 6) Most significant complication of gastric volvulus arises from vascular compromise to the stomach resulting in ischemic sequelae; 7) There is opportunity for collaborative management of patients utilizing both gastroenterology and general surgery; a less invasive approach using endoscopy for decompression and possible detorsion has been attempted followed by a more invasive approach of open reduction with or without gastropexy.

\section{Conclusions}

This case report describes a 21-year-old Hispanic female who presented with cholecystitis and subsequently underwent laparoscopic converted to open cholecystectomy. This patient had an extensively complicated postoperative course, ultimately resulting in the identification of a gastric volvulus that was secondary to adhesions from the original open cholecystectomy. This combination of inciting events has never been described before. Based upon the literature reviewed, gastric volvuli are most commonly associated with paraesophageal hernia without a specific predilection for gender. Similar to our patient, the most common symptoms associated with this disease are epigastric pain, nausea, vomiting, and distension. There is utility in using barium contrast study, upper gastrointestinal endoscopy, or CT for diagnosis of this condition. Once the diagnosis is made and the patient is hemodynamically stabilized, definitive treatment is achieved through open surgical reduction with or without gastropexy.

\section{Acknowledgments}

We would like to acknowledge RM for allowing us to share her story.

\section{Financial Disclosure}

None to declare.

\section{Conflict of Interest}

None to declare.

\section{Informed Consent}

Not applicable.

\section{Author Contributions}

$\mathrm{ME}$ and SD: report writing and editing; CR, DC, and JZ: report editing.

\section{Data Availability}

The authors declare that data supporting the findings of this study are available within the article. 


\section{References}

1. Carter R, Brewer LA, 3rd, Hinshaw DB. Acute gastric volvulus. A study of 25 cases. Am J Surg. 1980;140(1):99106.

2. Chau B, Dufel S. Gastric volvulus. Emerg Med J. 2007;24(6):446-447.

3. Costa MRP, Matos ASB, Almeida JR, Oliveira FJ. Primary gastric volvulus: a report of two cases. J Surg Case Rep. 2018;2018(8):rjy227.

4. Lopez P, Megha R. Gastric Volvulus. In: StatPearls. Treasure Island (FL): StatPearls Publishing; 2019.
5. Wasselle JA, Norman J. Acute gastric volvulus: pathogenesis, diagnosis, and treatment. Am J Gastroenterol. 1993;88(10):1780-1784.

6. Gourgiotis S, Vougas V, Germanos S, Baratsis S. Acute gastric volvulus: diagnosis and management over 10 years. Dig Surg. 2006;23(3):169-172.

7. Tsang TK, Walker R, Yu DJ. Endoscopic reduction of gastric volvulus: the alpha-loop maneuver. Gastrointest Endosc. 1995;42(3):244-248.

8. Rashid F, Thangarajah T, Mulvey D, Larvin M, Iftikhar SY. A review article on gastric volvulus: a challenge to diagnosis and management. Int J Surg. 2010;8(1):18-24. 